\title{
Hackathons as an Informal Learning Platform
}

\author{
Arnab Nandi \\ Computer Science \& Engineering \\ The Ohio State University \\ arnab@cse.osu.edu
}

\author{
Meris Mandernach \\ University Libraries \\ The Ohio State University \\ mandernach.1@osu.edu
}

\begin{abstract}
Hackathons are fast-paced events where competitors work in teams to go from an idea to working software or hardware within a single day or a weekend and demonstrate their creation to a live audience of peers. Due to the "fun" and informal nature of such events, they make for excellent informal learning platforms that attract a diverse spectrum of students, especially those typically uninterested in traditional classroom settings. In this paper, we investigate the informal learning aspects of Ohio State's annual hackathon events over the past two years, with over 100 student participants in 2013 and over 200 student participants in 2014. Despite the competitive nature of such events, we observed a significant amount of peer-learning - students teaching each other how to solve specific challenges and learn new skills. The events featured mentors from both the university and industry, who provided round-the-clock hands-on support, troubleshooting and advice. Due to the gamified format of the events, students were heavily motivated to learn new skills due to practical applicability and peer effects, rather than merely academic metrics. Some teams continued their hacks as long-term projects, while others formed new student groups to host lectures and practice building prototypes on a regular basis. Using a combined analysis of post-event surveys, student academic records and source-code commit log data from the event, we share insights, demographics, statistics and anecdotes from hosting these hackathons.
\end{abstract}

\section{INTRODUCTION}

Hackathons are events where computer programmers and others involved in software development, including graphic designers, interface designers and project managers, collaborate intensively on software projects in a short period of time, typically 24-36 hours. Traditionally software companies have held or sponsored these events to induce problem solving through creativity. Though hackathons have existed since the late 1990s, few have focused on emphasizing the key aspects of learning as a focal point of the event. A

Permission to make digital or hard copies of all or part of this work for personal or classroom use is granted without fee provided that copies are not made or distributed for profit or commercial advantage and that copies bear this notice and the full citation on the first page. Copyrights for components of this work owned by others than the author(s) must be honored. Abstracting with credit is permitted. To copy otherwise, or republish, to post on servers or to redistribute to lists, requires prior specific permission and/or a fee. Request permissions from permissions@acm.org.

SIGCSE '16, March 02 - 05, 2016, Memphis, TN, USA

(C) 2016 Copyright held by the owner/author(s). Publication rights licensed to ACM. ISBN 978-1-4503-3685-7/16/03 . .\$15.00

DOI: http://dx.doi.org/10.1145/2839509.2844590 university-sponsored hackathon gives developers an opportunity to compete, develop products, and potentially make important academic connections with faculty and labs. At Ohio State, the first university-wide, full-scale hackathon was held in November 2013 over a 24 hour period. There were 103 participants, constituting 34 teams of which 26 gave demonstrations of their work. Building on the success of the first year, in October 2014, a 36 hour hackathon featured over 200 participants comprising 59 teams.

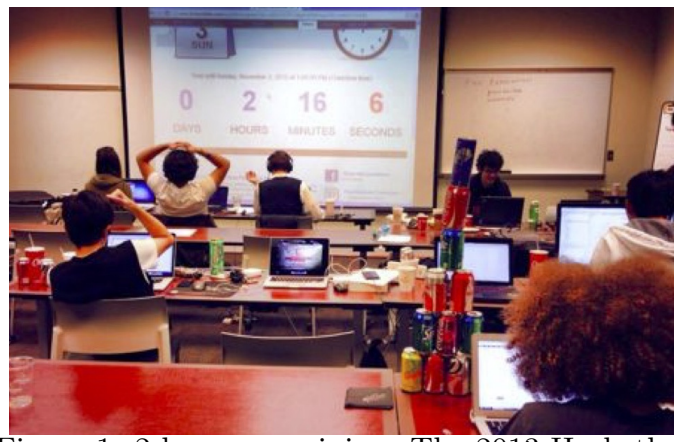

Figure 1: 2 hours remaining: The 2013 Hackathon

In 2013, projects built during the Hackathon ranged from an app to aid suicide prevention (a collaborative project built by a Psychology graduate student and 3 CSE students), to an alarm that read the weather, to a class selector for CSE that bundled class dependencies using clustering. In 2014, projects ranged from an app for checking into events from doctor appointments to class attendance; to a motorcyclemounted sensor data collection device.

\section{RELATED EFFORTS}

There is a tremendous amount of interest in making education more engaging and interesting to students. Beyond traditional curriculum, several programs have adopted project-based approaches [15] that allow students use a critical problem-solving approach while simultaneously integrating information from a variety of topical lectures and multiple disciplines. Though informal education $[6,9,17]$ has been considered an exciting and compelling way to get students introduced to Computer Science in higher education, the barrier to entry has always been relatively high because of the steep learning curve and specificity of concepts used in the discipline. This paper examines informal learning through a hackathon by analyzing team activity (e.g., commit histories), participant academic performance vs. nonparticipant performance (e.g., GPAs), and individual feedback from hackathon participants. 
Competitive Settings: One way to reduce barriers in the learning process is to shape education around competitive platforms. Johnson and Johnson [14] discussed several strategies of adapting pedagogy to include cooperative, competitive, and individualistic learning in the classroom. Competitive events [11] provide an excellent venue to facilitate learning since they simultaneously enforce a structure (i.e., rules of the competition) and allow for out-of-the-box and imaginative thinking. The introduction of gaming as a learning vehicle has also been studied heavily [26]. While coding competitions and hackathons $[10,23]$ have been considered before, the focus is typically entrepreneurial or industry driven [13], rather than learning outcomes. The hackathon model provides a fun and engaging format for students [1] akin to flipped classroom settings [22] and has been adapted to other disciplines [7] as well.

Industry-focused learning: Hackathons have been popular in industry settings $[5,23]$ and open source communities [8] - they are often seen as an efficient way to rapidly prototype [21] on ideas outside of the project schedule, or as a community exercise [4]. By designing aspects of the hackathon to focus on informal learning by and mentorship of students new opportunities emerge. Programs such as Yahoo! Inc.'s "Hack U" have brought industry partners into the university setting, allowing them an opportunity to both educate students about industry-relevant technologies and recruit students for internships or full-time positions [5]. For several industry partners, this is a rare opportunity to mentor and interview prospective employee candidates for an extended and intensive period of time.

Group and Peer Learning: Programming is often a solitary exercise and therefore it does not easily lend well to a cooperative, peer-based setting. However, for both graduate work and in industry, the ability to work in teams and leverage peer-assisted and self-directed learning are essential [3]. While the impacts of group learning, especially in computerbased education, have been well studied [12], specifically designing competitive events to exploit peer-learning techniques is challenging [24].

Addressing Diversity: As a discipline, Computer Science has faced a history of gender and diversity challenges [16]. Scragg and Smith [20] suggest that very few women even consider Computer Science as a possible major, and that efforts need to be made not just to retain, but to recruit students in to the field. Blum, et. al. [2] posit that the reasons for gender challenges include the environment and perception of the field. Thus, creating an appealing, collegial setting for women to learn new concepts without the formal and onerous requirements of coursework was a critical consideration when developing our hackathon events.

Libraries as Informal Learning Environments: Libraries have long served as a discipline neutral space where ideas can be presented and debated. In academic settings, libraries are spaces where learning can be both formal and informal. Students are able to meet and study and learn from each other or work independently and use it primarily as a study space. Walton and Matthews [25] point out that libraries are poised to contribute to the evaluation of informal learning spaces because librarians have the expertise and experience of developing spaces. More recently, libraries have evolved to support more non-traditional learning expe- riences by providing services related to makerspaces [19], $3 \mathrm{D}$ printing [18], hack spaces, and generally supporting the $D I Y$ (do-it-yourself) movement. As spaces evolve, so must library services. Libraries are thus primed to support the informal learning inherent in hackathons, and also have the required event infrastructure (e.g., wireless internet).

\section{THE ANATOMY OF A HACKATHON}

\subsection{Objectives and Event Format}

The primary goals of the hackathon were to raise awareness of technical talent at Ohio State and foster a competitive, yet cooperative, culture for talented individuals to showcase their knowledge. It also allowed participants to connect with faculty, labs, centers on campus, and most importantly, with each other. "We didn't know each other before we did this", said one team, who went from being strangers to working together over $20+$ hours and producing a demonstrable project.

The event format both years included a tech talk that kicked off the hackathon, a set period of hacking which included technical help from individuals from industry, followed by judging. The top teams were announced immediately following judging and public voting was conducted for crowd favorites. This was followed by an awards ceremony and networking event, allowing participants to showcase their projects in a science-fair format.

\subsection{Demographics}

For the 2013 event, there were 103 participants, constituting 34 teams, all from Ohio State. In 2014, this number roughly doubled to 202 participants in 59 teams. The opening up of the event to non-Ohio State students brought in 18 participants from other nearby universities: Bowling Green State University, Kent State University, University of Akron, University of Cincinnati, Wright State University and Youngstown State University. Based on a combination of registration data and student records, we present demographic information for the Ohio State participants below.

Gender: In terms of gender, there were 11 women participants in 2013. In 2014, this number increased to 30, i.e., $16 \%$. While this is roughly comparable to the representation of women in Computer Science, we plan to continue to address diversity and inclusion issues, as described in the previous sections.

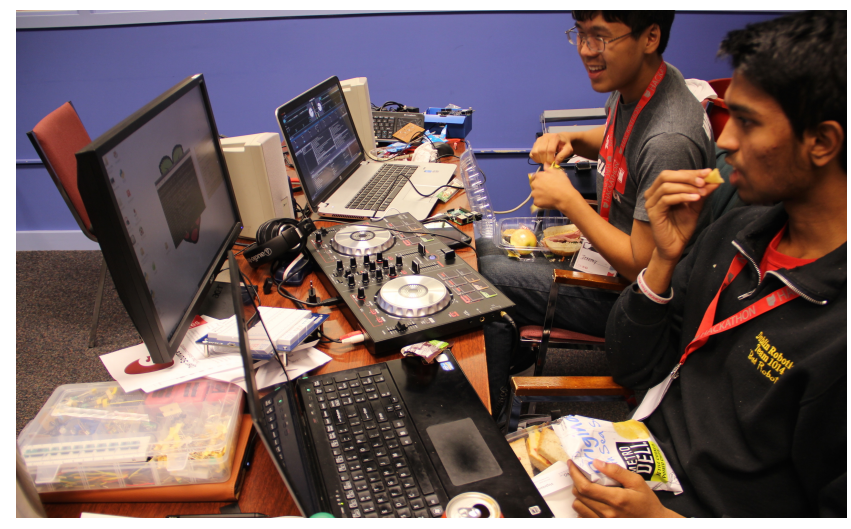

Figure 2: Diversity of disciplines: Hardware projects at the 2014 Hackathon 
Majors of Study: As shown in Table 1, while the event was predominantly Computer Science-focused, there were a considerable number of non-Computer Science majors who participated. Given that a workable output was expected from this hackathon, it was not surprising that Computer Science Engineering majors comprised the majority of the participants. However, as the popularity of the event grows, the number of non-Computer Science students is expected to rise, both through raising awareness of hackathons amongst the non-Computer Science communities, and also through affiliated domain-focused events, such as the $50+$ participant Makeathon conducted in April 2015 which focused on building hardware projects over a 24-hour period.

\begin{tabular}{|l|r|r|}
\hline Major & '13 (\%) & '14 (\%) \\
\hline Computer Science \& Engineering & 79 & 71 \\
\hline Electrical Engineering & 8 & 12 \\
\hline Physics \& Engineering Physics & 6 & 3.5 \\
\hline Mechanical Engineering & 1 & 3 \\
\hline Misc / Exploration & 1 & 3 \\
\hline Business & 0 & 2 \\
\hline Chemistry \& Chemical Engineering & 0 & 1 \\
\hline Biomedical & 0 & 1 \\
\hline Architecture \& Planning & 0 & 1 \\
\hline Geography & 2 & 0.5 \\
\hline Aero, Astronomy, \& Astrophysics & 2 & 0.5 \\
\hline Mathematics & 0 & 0.5 \\
\hline Civil Engineering & 0 & 0.5 \\
\hline Industrial Systems Engineering & 0 & 0.5 \\
\hline Psychology & 1 & 0 \\
\hline
\end{tabular}

Table 1: Demographics: Major

\begin{tabular}{|l|l|l|l|l|l|}
\hline Event & $1^{\text {st }} \mathrm{yr}$ & $2^{\text {nd }}$ yr & $3^{\text {rd }}$ yr & $4^{\text {th }} \mathrm{yr}$ & Grad \\
\hline $\mathbf{2 0 1 3}$ (\# students) & 6 & 19 & 22 & 33 & 23 \\
\hline $\mathbf{2 0 1 4}$ (\# students) & 12 & 33 & 37 & 78 & 24 \\
\hline
\end{tabular}

Table 2: Demographics: Year in School

Year of Study: The hackathon was a predominantly undergraduate focused, as shown in Table 2. However, there was a strong and significant representation of graduate students. Based on post-interviews and contributed resumes, the graduate students who participated had a history of involvement in competitive events during their undergraduate education. Some graduate students used the event to collaborate with individuals from other disciplines in order to bring their ideas to fruition, such as a psychology graduate student who partnered with CSE students to build a suicide prevention Android application. Other Computer Science graduate students used the hours as a focused way of building upon their existing research projects.

\subsection{How Teams Work}

In 2014, we introduced compulsory use of source control, sponsored by Github. Each team was required to save their code to a private repository on a regular basis, thereby promoting collaboration and responsible coding practices. Access to these repositories allowed for a unique opportunity: we could, on a fine-grained per-commit basis, discover insights on how 200 students collaboratively built software over a 36 -hour time period. We present our findings from analyzing the source code commit logs next, as shown in Figures 3-5. It should be noted that due to the scale and diversity of student teams, and since performance evaluations and surveys were anonymous, we use this analysis to gain insights and adapt our event to aid learning, and not as an absolute metric of student performance.

Self-pacing and Productivity: The extended nature of this event made pacing an important factor for productivity and learning. As described in the previous sections, we took care to provide resources and an initial orientation about taking care of themselves during such an event. However, in terms of the project, it was up to the participants to pace themselves and plan out how they would achieve their desired goals. In addition to qualitative and anecdotal data, the source code commit logs depicted noticeable patterns. As shown in Figures 4 and 5, productive teams kept a steady pace of progress throughout the event, saving code on a regular basis. In the 2013 event, we had noticed that several inexperienced teams gave up due to burnout. Feedback also indicated that an additional 12 hours would allow participants to sleep without worrying about completing their projects. The 36-hour format allowed students to take breaks and compete in a more relaxed environment. This is evidenced by the lull in activity of commits during the early hours of Saturday, when most students took a break after an initial flurry of coding activity, and returned the next day to continue their project.

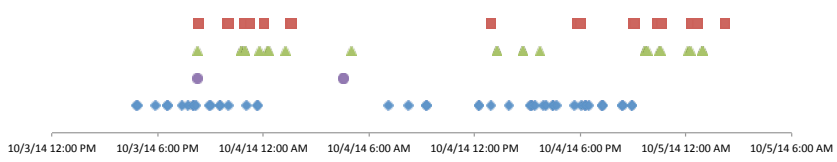

(a) Team taking turns to work, complementary commit patterns (relay model)

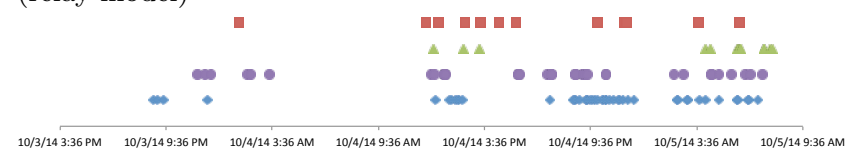

(b) Team working in coordinated schedules, taking breaks at the same time (waves model)

Figure 3: Models of collaboration over time (x-axis): each dot is a git commit from a team member (each student is represented by a different color / shape).

Different Models of Collaboration: Another interesting insight from the commit log data was that it evidenced the students teamwork. Two noticeable patterns of collaboration emerged. The first model (the relay approach, as seen in Figure 3a) involved teams taking turns working on the project. After an initial period of coordinated and simultaneous work, the team divided tasks, and one or two students took breaks while the other students in the team worked. One consideration to keep in mind is that not all productivity is immediately visible in the source code: e.g., if a student was acting as a project manager, or if two students were working in pairs / as a group, but using one computer. However, the log data shows this approach whereby the commits alternate between members of the group.

A contrasting approach used by other teams was the waves model (Figure 3b), where teams would meet on a frequent basis to work together and coordinate for brief periods, and then take breaks at the same time, or go off to work individually. Based on observations during the event, the particular team in Figure 3b was highly collaborative with all team members actively writing code, and used the source control system as a way to share code. 


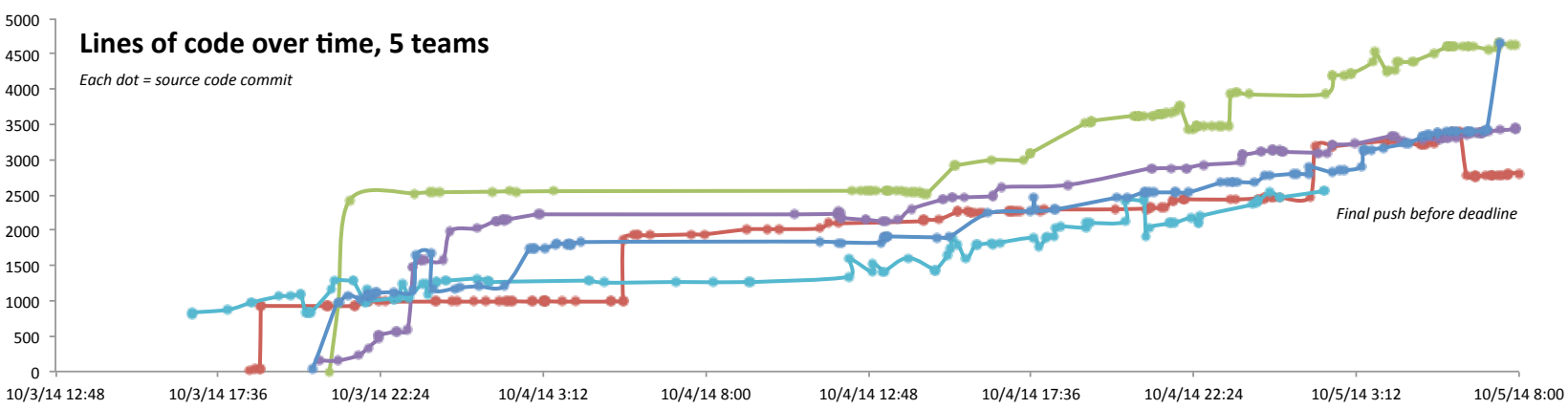

Figure 4: Projects being built over a 36 hour period: The x-axis represents time, and the y-axis represents number of lines of code. Each dot is a team member pushing their code to the repository.

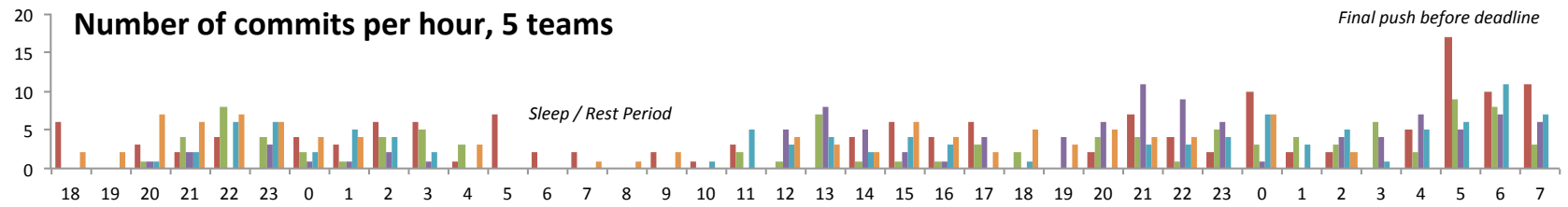

Figure 5: Number of commits over 36-hour Hackathon period.

In addition to individual team peer-to-peer learning, students regularly sought help from technical mentors, members of the Open Source Club, and other peers from other participating teams to figure out how to work past stumbling blocks during the events. Several individuals mentioned these approaches in their post-event surveys.

\section{POST-EVENT SURVEYS}

In addition to gathering feedback throughout the planning process, we conducted an anonymous post-event survey each year. Students rated the 2013 event at a $\mathbf{4 . 5}$ on $\mathbf{5}(20 \%$ response rate), and the 2014 event at $\mathbf{4 . 6}$ on $\mathbf{5}$ (36\% response rate). Drilling down to actual ratings, the 2014 event had a overwhelmingly positive rating: 50 students rated it a $\mathbf{5 / 5}$, and $\mathbf{9 1 \%}$ rated it at 4 or above. In addition to objective ratings, the surveys provided a lot of qualitative insight, which we have factored into our long-term planning.

On in-class vs practical learning: Due to the scale and diverse nature of the event, it is hard to objectively measure or correlate learning outcomes. However, as an initial insight, quotes from the students are highly encouraging:

- "Enjoyed the opportunity to build an application from start to finish. I learned a lot and truly understood how to apply my classroom/personal learnings."

- "Had a challenging but great weekend coding, really made my brain burn, which feels great."

- "It allowed me to participate and gain experience in a group programming setting, something that is not too stressed in my current/past classes."

- "Ability to create something useful and learn from others. Meeting more CSE students to collaborate with is always a positive."

- What I liked:] "The ability to sit down for an extended period of time and just work on a project allowed me to accomplish more in 24 hours than I have since the start of the school year and it made me feel like I actually did something useful for once."

- "I loved the fact that this event gave me time to learn how to implement real world applications of [computer science]."

On team skills: As organizers, we were ourselves very surprised at the level of teamwork. Some teams were formed at the event itself, while some other teams were formed during a "team match making" event organized by the ACM-W student group a week before the 2014 event.

- "I enjoyed working with a team to create the application. It was a good learning experience, because I had only just met my teammates."

- "I felt like I got a lot done and really accomplished something cool. I enjoyed working with my team. We will continue working on our project, so it helped us get off the ground."

- "What I liked was] The teamwork and collaboration between teams."

- "I loved being able to work in an environment with my peers."

- "I liked being around a lot of bright people. I was able to learn a lot, and I look forward to more hackathons."

On the availability of mentors: Having alumni come back to the University as technical mentors created the opportunity for them to be role models for students participating in the hackathon. They were able to help with experience and insights, e.g., providing advice into scoping projects conservatively due to the time limitations. We were pleasantly surprised by the participation levels of some mentors. Several used these opportunities to wander around and visit with hackathon participants, discuss jobs or internships at their companies, and provide hints to help solve pesky problems that arose in students' code. Many of the mentors were Ohio State alumni who work in the tech industry in the city and beyond. While mentors had initially committed to spending 4 hours with the students, some ended up spending $24+$ hours since they enjoyed the process.

- "It was a great opportunity to learn, meet peers, and network with professionals."

- "The motivation from the mentors was extremely helpful. The mentors themselves were awesome."

- "The availability of mentors was very nice, and they were for the most part approachable and helpful."

- "I enjoyed working with mentors as well as meeting people from other schools who had come to compete."

- "I really liked the energy of the people in the event as well as the organization of the mentors within a structured setup. The best part was the free food that was given all weekend, which tied with the excellent help and support given by the mentors." 
- A first time participant noted: "While working on a project with friends was fun, and I learned a lot from it, the chance to talk to other like-minded people and meeting mentors was definitely one of the most invaluable experiences from the event."

- "Awesome mentors - they really made it for me."

\section{ACADEMIC PERFORMANCE}

In Table 3, we present a term-by-term analysis of $\mathrm{Cu}$ mulative and Term GPAs for Undergraduate students during the existence of our program. (Some numbers / measures are withheld for institutional privacy and administrative reasons.) While we do not expect a single weekend event each year to have a measurable impact on a student's performance, does the long-term impact on student morale, community, self-confidence and peer-learning have a bearing on academic performance? One concern is that the sustained time involvement of such extracurricular events compete with homework and class projects, and are a timesink in a student's already busy academic schedule: they may have a negative impact on in-class performance. In our analysis of student grades, we found this concern to be unwarranted: our analysis revealed that the hackathonparticipating students have small $(2-5 \%)$ but consistently higher GPAs than non-participating students. When comparing performance in individual to see if students who participated in the Hackathons got better, worse, or stayed roughly the same from one term to the next ("better" / "worse" defined as a GPA increase / decrease of 0.1 or more), we were unable to find any significant patterns. Thus, while we cannot claim that the Hackathon causes or impacts better GPAs, our conjecture is that such activities seem to consistently self-select a higher achieving group of students.

There are two ideal followups to this analysis: a cohort study isolating student "profiles" by analyzing not just averages, but the distribution of GPAs in each of these groups. Second, a large-scale, longitudinal study tracking the entirety of a student's progress through each term in college would allow us to control for a variety of possible impactors - these are ideal future work.

\section{LEARNING OUTCOMES}

Industry Relevance: Both judges and mentors from local and national industry participated in the events. They were able to use these events to network and recruit students from Ohio State. They also were able to make valuable connections with each other as they waited for students to approach them and ask questions. This way, sponsors have two potential outcomes of the event: recruitment of current students and engaging with colleagues from other companies.

The planners of the hackathon included several student organizations. Through these student organizations several events happened prior to the hackathon and served to market the event to potential attendees as well as facilitate the organization of teams, teach students the basics of GitHub commits, as well as coordinate tech talks from industry sponsors. These individuals learned valuable lessons related to project management, key details of navigating the infrastructure of higher education, reaching out to local news agencies, and soliciting sponsorships from vendors.

Long-term projects: There were several excellent examples of long-term projects that germinated at these events. Our source code repository showed signs of activity days,

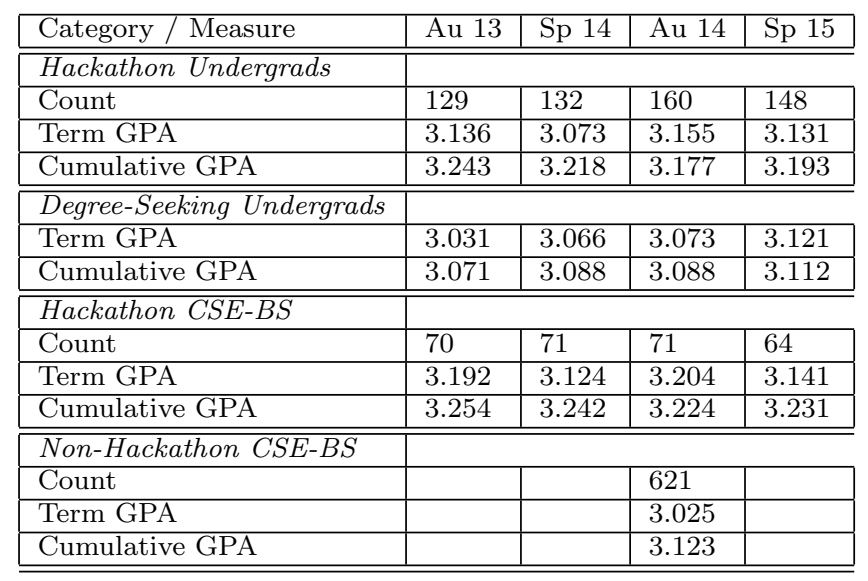

Table 3: Average Cumulative and Term GPAs for Undergraduate students. Hackathon-participating students have small $(2-5 \%)$ but consistently higher GPAs than nonparticipating students.

and even weeks after the Hackathon - a sign that students were working on their projects well after they were due. One group that did not make it to the top ten projects at the awards ceremony continued working on their project and received undergraduate research scholarships that let them complete their project in the following semester. In the longer term, we additionally expect to see entrepreneurial activity based on both the projects prototyped and social connections developed during the event.

Long-term team skills: One particularly interesting observation was a team comprising 4 students, each from a different university. Through in-person conversations and further followups, we learned that the team went to the same high school, and they used university hackathon events such as ours to reconnect and collaborate using skills learned at different institutions.

\section{ANCILLARY OUTCOMES}

Industry-facing Skills: Yen et al. [27] suggest that skills most needed by information systems professionals in industry include interpersonal behavior and communication skills, personal motivation and working independently, and critical and creative thinking. These are exactly the skills developed through events such as hackathons. This informal learning and skill development better equips students to market themselves for internships and jobs after college.

All of the mentors and judges who participated in the hackathons mentioned that they enjoyed the large amount of face-to-face time they had with students. They were able to use the mentoring opportunities as an extended interview and really understood the capabilities of the students after working with them through the course of the hackathon. Additionally, university-industry collaboration is always time-intensive because project-development and relationship-building are iterative and complex. Using the hackathon format to develop work strategies allows industry sponsors to jumpstart productivity on specific projects and by providing mentors they're able to iterate quickly with the hackathon participants.

In-University Benefits: In addition to the specific benefits of creating a tech culture at Ohio State University, there were some unforeseen benefits that have arisen from 
the hackathon events. Several students enrolled into courses such as "Mobile app development" or specific capstones after attending the event, demonstrating the promise of the Hackathon serving as both an on-ramp into a university's course offerings, and also a venue to demonstrate skills learned from these courses. From the 2013 event, one team developed an app to train for a large charity bike ride and they were contacted by that organization and hired to continue their hackathon project. One key benefit for the university and for students is that students are able to be recruited from this event for specific research assistantships. Most notably, students were contacted by Computer Science faculty and recruited to be part of labs. Though few of the teams chose to work on staff-suggested projects, one plan for the future is to advertise projects earlier and market them to students as potential job leads should they choose to work on the projects. This allows university units to leverage the hackathon as source of creativity for those who need help specific developing apps or web interfaces to augment their domain-specific research.

Future Events: The interest from the events has led to the formation of the "Buckeye Hackers Club", with an active membership of over 40 individuals. Funds, insights, and infrastructure from the hackathon events were used to enable other related events, such as TownHack, a city and community-focused hackathon (Nov 2014), and the Electronics Club-hosted Makeathon hardware hackathon (April 2015). Planning for the 2015 hackathon is underway.

\section{CONCLUSION}

The hackathons held at Ohio State have provided a structure for increased informal learning. They have spawned creativity and have resulted in continued enthusiasm amongst students, and established valuable mentorship connections with alumni. Given the time constraints of the competition, students are able to quickly teach and learn from their peers (not just teammates) in order to produce a workable product in time for the showcase. Students learn to compress their development process into a short time frame, allowing them to hone their problem solving, project management, and prioritization skills - all while still maintaining a fun, enthusiastic, and engaging experience. In addition to surveys and activity information from the event itself, additional data indicates that students participating in a Hackathon tend to have stronger GPA than their peers.

What started as an idea to host a single hackathon has blossomed into a complete program dedicated towards creating informal settings for learning in a variety of different areas. The program is now sponsoring students to attend other hackathons, funding related events, and soliciting greater partnerships with industry. These initiatives focus on fostering an inclusive tech culture focused on informal learning (both individual and peer-based) and higher levels of engagement with the technology industry.

Acknowledgements: The first author was supported in part through U.S. National Science Foundation CAREER award \#1453582. We would like to thank Christopher Wallace, Matt Faluotico, Elizabeth Burl, George Kvaratskhelia, Barry Brinkley, Eric Schnell, Daniel Dotson, Charlene Brenner, Krysta Kirsch, Robert VanDyne, Linda Katunich, Swaroop Joshi, Neelam Soundarajan, Paulo Sivilotti, Rajiv Ramnath for providing support, data, and valuable feedback towards both the paper and the events.

\section{REFERENCES}

[1] J. A. Artiles and D. R. Wallace. Borrowing from Hackathons: Overnight Designathons as a Template for Creative Idea Hubs. WEEF, 2013.

[2] L. Blum, C. Frieze, et al. A Cultural Perspective on Gender Diversity in Computing. SIGCSE, 2006.

[3] D. Boud, R. Cohen, and J. Sampson. Peer Learning in Higher Education: Learning from and With Each Other. Routledge, 2014.

[4] D. Boud and H. Middleton. Learning from Others at Work: Communities of Practice and Informal Learning. Journal of Workplace Learning, 2003.

[5] G. Briscoe and C. Mulligan. Digital Innovation: the Hackathon Phenomenon. Creative Works, 2014.

[6] R. R. Burton and J. S. Brown. An Investigation of Computer Coaching for Informal Learning Activities. International Journal of Man-Machine Studies, 1979.

[7] M. Calco and A. Veeck. The Markathon: Adapting the Hackathon Model for an Introductory Marketing Class Project. Marketing Education Review, 2015.

[8] L. Christopherson et al. Developing Scientific Software Through the Open Community Engagement Process. Technical Report, 2013.

[9] F. Coffield. The Necessity of Informal Learning. Policy Press, 2000.

[10] K. Goetz. Hackathon: 2 Days, 1,000 Developers, Lots of Caffeine. All Things Considered, 2010.

[11] J. Grimes et al. Robotics Competition: Providing Structure, Flexibility, and an Extensive Learning Experience. FIE, 2008.

[12] S. Hooper. Effects of Group Composition on Achievement, Interaction \& Learning Efficiency. ETRD, 1991.

[13] L. Irani. Hackathons and the Making of Entrepreneurial Citizenship. Science, Technology \& Human Values, 2015.

[14] D. W. Johnson et al. Learning Together 63 Alone: Cooperative, Competitive, $\&$ Individualistic Learning. Prentice-Hall, 1987.

[15] J. J. Kellar, W. Hovey, et al. A Problem Based Learning Approach for Freshman Engineering. FIE, 2000.

[16] J. Margolis, A. Fisher, and F. Miller. The Anatomy of Interest: Women in Undergraduate Computer Science. Women's Studies Quarterly, 2000.

[17] V. J. Marsick and K. E. Watkins. Informal and Incidental Learning. New Directions for Adult and Continuing Education, 2001.

[18] H. Michele Moorefield-Lang. Makers in the Library: Case Studies of 3D Printers and Maker Spaces in Library Settings. Library Hi Tech, 2014.

[19] A. Pashia. Empty Bowls in the Library Makerspaces Meet Service. College $\&$ Research Libraries, 2015.

[20] G. Scragg and J. Smith. A Study of Barriers to Women in Undergraduate Computer Science. SIGCSE Bulletin, 1998.

[21] A. Sigfridsson, G. Avram, A. Sheehan, and D. K. Sullivan. Sprint-driven Development: Working, Learning and the Process of Enculturation in the PyPy Community. Open Source Development, Adoption and Innovation, 2007.

[22] M. Skirpan and T. Yeh. Beyond the Flipped Classroom: Learning by Doing Through Challenges and Hack-a-thons. SIGCSE, 2015.

[23] S. Sousa. Lessons learnt from a public-private big data hackathon. Big Innovation Centre Report, 2013.

[24] K. J. Topping. Trends in Peer Learning. Educational Psychology, 2005.

[25] G. Walton and G. Matthews. Evaluating University's Informal Learning Spaces: Role of the University Library? New Review of Academic Librarianship, 2013.

[26] U. Wolz, T. Barnes, I. Parberry, and M. Wick. Digital Gaming as a Vehicle for Learning. SIGCSE Bulletin, 2006.

[27] D. C. Yen, S. Lee, and S. Koh. Critical Knowledge/Skill Sets Required by Industries: an Empirical Analysis. Industrial Management \& Data Systems, 2001. 